\title{
ON THE DIMENSION OF DISCRETE VALUATIONS OF $k\left(\left(X_{1}, \ldots, X_{n}\right)\right)$
}

\author{
Miguel Ángel Olalla Acosta * \\ Departamento de Álgebra. Universidad de Sevilla, España.
}

\begin{abstract}
Let $v$ be a rank-one discrete valuation of the field $k\left(\left(X_{1}, \ldots, X_{n}\right)\right)$. We know, after [1], that if $n=2$ then the dimension of $v$ is 1 and if $v$ is the usual order function over $k\left(\left(X_{1}, \ldots, X_{n}\right)\right)$ its dimension is $n-1$. In this paper we prove that, in the general case, the dimension of a rank-one discrete valuation can be any number between 1 and $n-1$.
\end{abstract}

\section{TERMINOLOGY AND PRELIMINARIES}

Let $k$ be an algebraically closed field of characteristic $0, R_{n}=k \llbracket X_{1}, \ldots, X_{n} \rrbracket$, $M_{n}=\left(X_{1}, \ldots, X_{n}\right)$ the maximal ideal and $K_{n}=k\left(\left(X_{1}, \ldots, X_{n}\right)\right)$ the quotient field. Let $v$ be a rank-one discrete valuation of $K_{n} \mid k, R_{v}$ its valuation ring, $\mathfrak{m}_{v}$ its maximal ideal and $\Delta_{v}$ its residual field of $v$. The center of $v$ in $R_{n}$ is $\mathfrak{m}_{v} \cap R_{n}$. Throughout this paper "discrete valuation of $K_{n} \mid k$ " will mean "rankone discrete valuation of $K_{n} \mid k$ whose center in $R_{n}$ be the maximal ideal $M_{n}$ ". The dimension of $v$ is the transcendence degree of $\Delta_{v}$ over $k$. We shall suppose that the group of $v$ is $\mathbb{Z}$.

Let $\widehat{K}_{n}$ be the completion of $K_{n}$ with respect to $v$ (see [2]), $\widehat{v}$ the extension of $v$ to $\widehat{K}_{n}, R_{\widehat{v}}, \mathfrak{m}_{\widehat{v}}$ and $\Delta_{\widehat{v}}$ the ring, maximal ideal and the residual field of $\widehat{v}$, respectively.

\section{THE DIMENSION OF $v$}

Fix a number $m$ between 1 and $n-1$. We are featuring a constructive method in order to obtain examples of valuations with dimension $m$.

\footnotetext{
*Partially supported by Junta de Andalucía, Ayuda a grupos FQM 218.
} 
Let us consider the following homomorphism:

$$
\begin{aligned}
\varphi: k \llbracket X_{1}, \ldots, X_{n} \rrbracket & \longrightarrow \overline{k(u) \llbracket t \rrbracket} \\
X_{1} & \longmapsto t \\
X_{2} & \longmapsto u t \\
X_{i} & \longmapsto \sum_{j \geq 1} u^{1 / p_{i}^{j}} t^{j}
\end{aligned}
$$

where $\overline{k(u)}$ stands for the algebraic closure of $k(u)$ and $2<p_{3}<\ldots<p_{n}$ are prime numbers.

Lemma 1 The homomorphism $\varphi$ is one to one.

Proof: Let us take the fields $K_{2}=k(u)$ and

$$
K_{i}=k\left(u,\left\{u^{1 / p_{3}^{j}}, j \geq 1\right\}, \ldots,\left\{u^{1 / p_{i}^{j}}, j \geq 1\right\}\right)
$$

for all $i \geq 3$.

Let us suppose that $\varphi$ is not one to one, then $\operatorname{ker}(\varphi) \neq\{0\}$. So let $f$ be a non-zero element of $M=\left(X_{1}, \ldots, X_{n}\right)$ such that $f \in \operatorname{ker}(\varphi)$. Let $m$ be the higher index such that $f \in k \llbracket X_{1}, \ldots, X_{m} \rrbracket$.

If $\underline{m=1 \text { or } 2}$, trivially we have a contradiction.

If $\underline{m=3}$, let us take

$$
\bar{f}=f\left(\varphi\left(X_{1}\right), \varphi\left(X_{2}\right), X_{3}\right) \in K_{2} \llbracket t, X_{3} \rrbracket,
$$

and consider the homomorphism

$$
\begin{aligned}
\psi: K_{2} \llbracket t, X_{3} \rrbracket & \longmapsto \overline{k(u) \llbracket t \rrbracket} \\
t & \longmapsto t \\
X_{3} & \longmapsto \sum_{j \geq 1} u^{1 / p_{3}^{j}} t^{j}
\end{aligned}
$$

We know that $\bar{f} \in \operatorname{ker}(\psi)$ and this kernel is a prime ideal because $\psi$ is an homomorphism between integral domains. We can write $\bar{f}=t^{r} g$, with $r \geq 0$ and $t$ doesn't divide to $g$. This forces $g$ to have some non-trivial terms in $X_{3}$. Let $s>0$ be the minimum such that $\alpha X_{3}^{s}$ is one of these terms. By the Weierstrass preparation theorem we have $g=U g^{\prime}$, where $U\left(t, X_{3}\right)$ is a unit and

$$
g^{\prime}=X_{3}^{s}+a_{1}(t) X_{3}^{s-1}+\ldots+a_{s}(t) .
$$

Since $U$ is a unit, $g^{\prime} \in \operatorname{ker}(\psi)$ and

$$
\psi\left(g^{\prime}\right)=g^{\prime}\left(t, \sum_{j \geq 1} u^{1 / p_{3}^{j}} t^{j}\right)=0 .
$$

This leads to a contradiction because the roots of $g^{\prime}$ are in $K_{2} \llbracket t^{1 / q} \rrbracket$, with $q \in \mathbb{Z}$, by the Puiseux theorem. 
If $\underline{m>3}$ let us take

$$
\bar{f}=f\left(\varphi\left(X_{1}\right), \ldots, \varphi\left(X_{m-1}\right), X_{m}\right) \in K_{m-1} \llbracket t, X_{m} \rrbracket
$$

and consider the homomorphism

$$
\begin{aligned}
\psi: K_{m-1} \llbracket t, X_{m} \rrbracket & \longmapsto \overline{k(u) \llbracket t \rrbracket} \\
t & \longmapsto t \\
X_{m} & \longmapsto \sum_{j \geq 1} u^{1 / p_{m}^{j}} t^{j} .
\end{aligned}
$$

As in the previous case we can write $\bar{f}=t^{r} h$, where $h \in \operatorname{ker}(\psi)$. So we have $h=U h^{\prime}$, where $U\left(t, X_{m}\right)$ is a unit and

$$
h^{\prime}=X_{m}^{r}+b_{1}(t) X_{m}^{r-1}+\ldots+b_{r}(t) \in \operatorname{ker}(\psi),
$$

so

$$
h^{\prime}\left(t, \sum_{j \geq 1} u^{1 / p_{m}^{j}} t^{j}\right)=0 .
$$

But this is again a contradiction by the Puiseux theorem: since $\operatorname{ker}(\psi)$ is a prime ideal, we can suppose that $h^{\prime}$ is an irreducible element of the ring $K_{m-1} \llbracket t \rrbracket\left[X_{m}\right]$. In this situation the Puiseux theorem says that to obtain the coefficients of a root of $h^{\prime}=0$, like a Puiseux series in $t$ with coefficients in $k(u)$, we have to resolve a finite number of algebraic equations of degree greater than 1 in $K_{m-1}$. Inside $K_{m-1}$ we can not obtain $u^{1 / p_{m}}$ and, with a finite number of algebraic equations, we can obtain a finite number of powers of $u^{1 / p_{m}^{j}}$ but not all. So this proves the lemma.

We shall extend to the quotient fields this injective homomorphism for giving an example of a rank-one discrete valuation of $k\left(\left(X_{1}, \ldots, X_{n}\right)\right)$ of dimension 1 .

Lemma 2 There exists a rank-one discrete valuation of $k\left(\left(X_{1}, \ldots, X_{n}\right)\right)$ of $d i$ mension 1.

Proof: We know that the homomorphism

$$
\varphi: k \llbracket X_{1}, \ldots, X_{n} \rrbracket \rightarrow \overline{k(u)} \llbracket t \rrbracket
$$

previously defined is one to one. So we can take the valuation $v=\nu \circ \varphi$, where $\nu$ is the usual order function over $\overline{K(u)}((t))$ in $t$ and $\varphi$ is the natural extension to the quotient fields. Let $\alpha$ be the residue $X_{2} / X_{1}+\mathfrak{m}_{v} \in \Delta_{v}$. Hence, to obtain the lemma we have to prove that $\alpha \notin k$ and $\Delta_{v}$ is an algebraic extension of $k(\alpha)$.

Let us suppose that $\alpha \in k$. Then there must exist $a \in k$ such that $X_{2} / X_{1}+$ $\mathfrak{m}_{v}=a+\mathfrak{m}_{v}$, so

$$
\frac{X_{2}-a X_{1}}{X_{1}} \in \mathfrak{m}_{v}
$$


This means that $v\left(X_{2}-a X_{1}\right)>1$. On the other side we have

$$
\varphi\left(X_{2}-a X_{1}\right)=(u-a) t
$$

so $v\left(X_{2}-a X_{1}\right)=1$ and we have a contradiction. Hence $\alpha \notin k$.

Let us prove that $\Delta_{v}$ is an algebraic extension of $k(\alpha)$. We can consider each element of $k \llbracket X_{1}, \ldots, X_{n} \rrbracket$ like a sum of forms with respect to the usual degree. If $f_{r}$ is a form of degree $r$, then $\varphi\left(f_{r}\right)=t^{r} P$, with $P$ a polynomial in $u$ and a finite number of elements $u^{1 / p_{i}}$.

Let us take $f, g \in k \llbracket X_{1}, \ldots, X_{n} \rrbracket$ such that $g \neq 0$ and $v(f / g)=0$. Then $\varphi(f / g)=h_{0}+t h_{1}$, where $h_{0}$ is a rational fraction in $u$ and a finite number of elements $u^{1 / p_{i}}$. So $h_{0}$ is algebraic over $k(u)$. Let us consider

$$
P(u, Z)=c_{0}(u) Z^{m}+c_{1}(u) Z^{m-1}+\ldots+c_{m-1}(u) Z+c_{m}(u) \in k[u][Z]
$$

a polynomial satisfied by $h_{0}$, where $c_{i}(u) \in k[u]$ for all $i$ and $c_{0} \neq 0$. Let $\beta$ be the element

$$
\beta=P\left(\frac{X_{2}}{X_{1}}, \frac{f}{g}\right)=c_{0}\left(\frac{X_{2}}{X_{1}}\right)\left(\frac{f}{g}\right)^{m}+\ldots+c_{m}\left(\frac{X_{2}}{X_{1}}\right) .
$$

Then we have

$$
\varphi(\beta)=c_{0}(u)\left(h_{0}+t h_{1}\right)^{m}+\ldots+c_{m}(u),
$$

so $v(\beta)=\nu \circ \varphi(\beta)>0$ and $\beta \in \mathfrak{m}_{v}$. Subsequently,

$$
0+\mathfrak{m}_{v}=\beta+\mathfrak{m}_{v}=P\left(\alpha, \frac{f}{g}+\mathfrak{m}_{v}\right) .
$$

This proves that $f / g+\mathfrak{m}_{v}$ is an algebraic element over $k(\alpha)$ and, a fortiori, the lemma.

Lemma 3 The dimension of a rank-one discrete valuation of $k\left(\left(X_{1}, \ldots, X_{n}\right)\right)$ is between 1 and $n-1$.

Proof: We know, after [1], that the dimension of a rank-one discrete valuation of $k\left(\left(X_{1}, \ldots, X_{n}\right)\right)$ is minor or equal than $n-1$. So we have to prove that there exists a transcendental residue in $\Delta_{v}$.

Let us suppose that $v\left(X_{i}\right)=n_{i}$ for all $i=1, \ldots, n$. Then the value of $X_{2}^{n_{1}} / X_{1}^{n_{2}}$ is zero, so $0 \neq\left(X_{2}^{n_{1}} / X_{1}^{n_{2}}\right)+\mathfrak{m}_{v} \in \Delta_{v}$. If this residue lies in $k$ then there exists $a_{21} \in k$ such that

$$
\frac{X_{2}^{n_{1}}}{X_{1}^{n_{2}}}+\mathfrak{m}_{v}=a_{21}+\mathfrak{m}_{v}
$$

This implies

$$
\frac{X_{2}^{n_{1}}}{X_{1}^{n_{2}}}-a_{21}=\frac{X_{2}^{n_{1}}-a_{21} X_{1}^{n_{2}}}{X_{1}^{n_{2}}} \in \mathfrak{m}_{v}
$$


and then

$$
v\left(\frac{X_{2}^{n_{1}}-a_{21} X_{1}^{n_{2}}}{X_{1}^{n_{2}}}\right)>0 .
$$

So we have $v\left(X_{2}^{n_{1}}-a_{21} X_{1}^{n_{2}}\right)=m_{1}>n_{1} n_{2}$. Then

$$
v\left(\frac{\left(X_{2}^{n_{1}}-a_{21} X_{1}^{n_{2}}\right)^{n_{1}}}{X_{1}^{m_{1}}}\right)=0 .
$$

If the residue of this element lies too in $k$, then there must exist $a_{22} \in k$ such that $v\left(\left(X_{2}^{n_{1}}-a_{21} X_{1}^{n_{2}}\right)^{n_{1}}-a_{22} X_{1}^{m_{1}}\right)=m_{2}>n_{1} m_{1}$. We can repeat this operation.

The previous procedure is finite: if it didn't stop we would construct the power series

$$
X_{2}^{n_{1}}-\sum_{i=1}^{\infty} b_{2 i} X_{1}^{i}
$$

such that the sequence of partial sums has increasing values. Since $\widehat{K_{n}}$ is a complete field, then this series amounts to zero in contradiction with $X_{1}$ and $X_{2}$ being formally independent. So the procedure must stop and there exists a transcendental element over $k$ in $\Delta_{v}$.

Theorem 4 Let $m$ be a fixed number between 1 and $n-1$, then there exists a rank-one discrete valuation of $k\left(\left(X_{1}, \ldots, X_{n}\right)\right)$ of dimension $m$.

Proof: Let us consider the one to one (the proof of injectivity parallels that of lemma 1) homomorphism

$$
\begin{aligned}
& \varphi \quad k \llbracket X_{1}, \ldots, X_{n} \rrbracket \quad \longrightarrow \quad \overline{k(u)} \llbracket t_{1}, \ldots, t_{m} \rrbracket \\
& X_{1} \longmapsto t_{1} \\
& X_{2} \longmapsto u t_{1} \\
& X_{i} \longmapsto\left\{\begin{array}{l}
t_{i} \text { if } i \leq m+1 \\
\sum_{j \geq 1} u^{1 / p_{i}^{j}} t_{1}^{j} \text { if } i>m+1 .
\end{array}\right.
\end{aligned}
$$

We can take the valuation $v:=\nu \circ \varphi$, with $\nu$ the usual order function in $\overline{k(u)} \llbracket t_{1}, \ldots, t_{m} \rrbracket$ and $\varphi$ the natural extension to the quotient fields. We know (lemma 2) that the residue $X_{2} / X_{1}+\mathfrak{m}_{v}$ is transcendental over $k$. Trivially the residue $X_{i} / X_{1}+\mathfrak{m}_{v}$ for all $i=3, \ldots, m+1$ are transcendental over $k\left(X_{2} / X_{1}+\right.$ $\left.\mathfrak{m}_{v}, \ldots, X_{i-1}+\mathfrak{m}_{v}\right)$ because $t_{i}$ are formally independent variables. Any element $f / g+\mathfrak{m}_{v} \in \Delta_{v}$ is algebraic over $k\left(X_{2} / X_{1}+\mathfrak{m}_{v}, \ldots, X_{m+1}+\mathfrak{m}_{v}\right)$ parallels that of lemma 2. So the dimension of $v$ is $m$.

\section{References}

[1] E. Briales, Constructive theory of valuations., Comm. Algebra 17 (1989), no. $5,1161-1177$.

[2] J. P. Serre, Corps locaux., Hermann, Paris, 1968. 